\title{
Acromegaly Presenting As Cardiac Failure - A Case Report
}

\author{
Shohael Mahmud Arafat ${ }^{1}$, Mahammad Abul Kalam Azad ${ }^{2}$, Rezwanur Rahman ${ }^{3}$, Mehruba Alam Ananna ${ }^{4}$, Ahmed \\ Manzurul Aziz ${ }^{5}$, Quazi Mamtazuddin Ahmed ${ }^{6}$, Muhammad Khaled Hasan ${ }^{7}$, ABM Abdullah $^{8}$
}

${ }^{1}$ Associate Professor, ${ }^{2}$ Medical officer, ${ }^{3}$ Resident, Department of Medicine, BSMMU, Dhaka. ${ }^{4}$ egistrar , BIRDEM ${ }^{5}$ Medical Officer, Singair Health Complex ${ }^{6}$ Assistant Professor, Department of Medicine, ${ }^{7}$ Medical officer. Department of Neurolgy, ${ }^{8}$ Professor and Dean, Department of Medicine, BSMMU, Dhaka.

\begin{abstract}
:
Acromegaly is characterized by chronic hypersecretion of growth hormone (GH) and is associated with increased mortality rate because of the potential complications such as cardiovascular disease, respiratory disease, or malignancy, which are probably caused by the long-term exposure of tissues to excess GH, for at least 10 years, before diagnosis and treatment. Here we are reporting a case of acromegaly who initially presented with features of left ventricular failure for which she got herself admitted in CCU and was treated conservatively. Later on, after clinical examination and investigations she was diagnosed as a case of mitral regurgitation due to cardiomyopathy caused by acromegaly. After the successful transsphenoidal resection of the pituitary microadenoma, the level of GH was normalized and heart failure improved.
\end{abstract}

Key words: acromegaly, heart failure, Pituitary microadenoma.

[BSMMU J 2011; 4(2): 122-124]

\section{Introduction:}

Patients with acromegaly have been reported to have about $30 \%$ higher mortality rate, and cardiovascular disease accounts for $60 \%$ of the deaths. We are reporting a case of a patient with acromegaly who was diagnosed with severe cardiac failure at the time of diagnosis and had marked clinical improvement after the successful resection of the pituitary adenoma. Immediate diagnosis and treatment are required for better control of acromegalic heart failure.

\section{Case Report:}

A 70 years old lady presented with a short history of dyspnoea (NYHA IV). On query she admitted that she noticed progressive enlargement of acral parts, coarsening and enlargement of face for 7 years, orthopnoea and palpitation for 3 months and new onset of diabetes mellitus and hypertension for 1 month . She also had excessive sweating and knee joint arthalgia but gave no history of headache, visual impairment, nasal discharge or any limb weakness. Clinical examination revealed acromegalic facies, enlarged acral parts, thyromegaly, features of cardiomegaly with mitral regurgitation, controlled B.P (with medication), and husky voice. Fundus examination and visual evaluation were unremarkable. Serum GH level was $15.0 \mathrm{ng} / \mathrm{ml}$ at basal level, and serum prolactin, TSH, $\mathrm{FT}_{4}$ were within normal limit. X-ray skull showed apparently normal size sella without any erosion but MRI of pituitary gland showed pituitary microadenoma. X ray hand showed increased soft tissue shadow with enlargement of hand bones with tufting of terminal phalanges with periarticular osteopenia. $\mathrm{X}$ ray

Address of correspondence: Dr Shohael Mahmud Arafat, Dept of Medicine,BSMMU, Dhaka E mail: arafatdr@yahoo.com foot shows increased heel pad thickness. CXR revealed cardiomegaly with scoliosis. Echocardiography showed systolic dysfunction with E.F 40\% with moderate mitral regurgitation. After confirmation of the diagnosis of acromegaly due to pituitary microadenoma transsphenoidal endoscopic pituitary surgery was done following stabilization of cardiac function. After successful surgery tissue was sent for histopathology which showed pituitary neoplasm composed of fairly uniform polygonal cells arranged in sheets. The cells had abundant eosinophilic cytoplasm and supporting connective tissue stroma was scarce. Post operative growth hormone level came down to normal with uneventful postoperative recovery. The patient was discharged at home on $9^{\text {th }}$ post operative day.

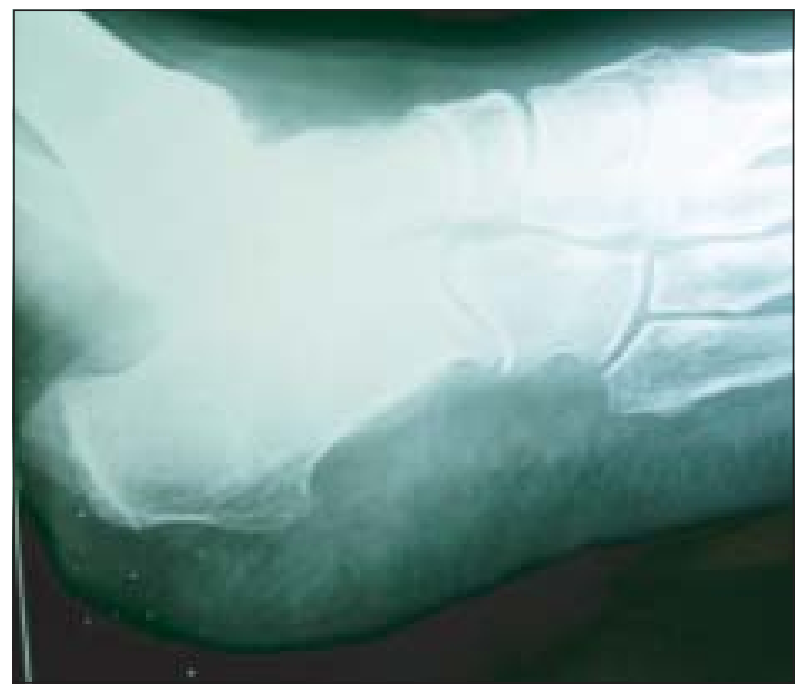

Fig.-1:X ray foot shows increased heel pad thickness. 


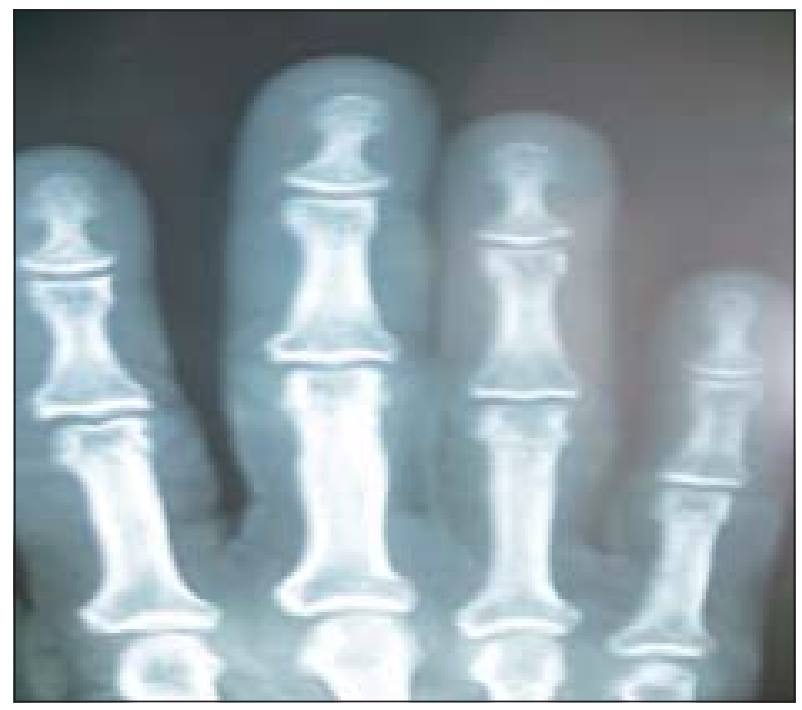

Fig.-2: $X$ ray hand showing increased soft tissue shadow with bony enlargement and tufting of terminal phalanges.

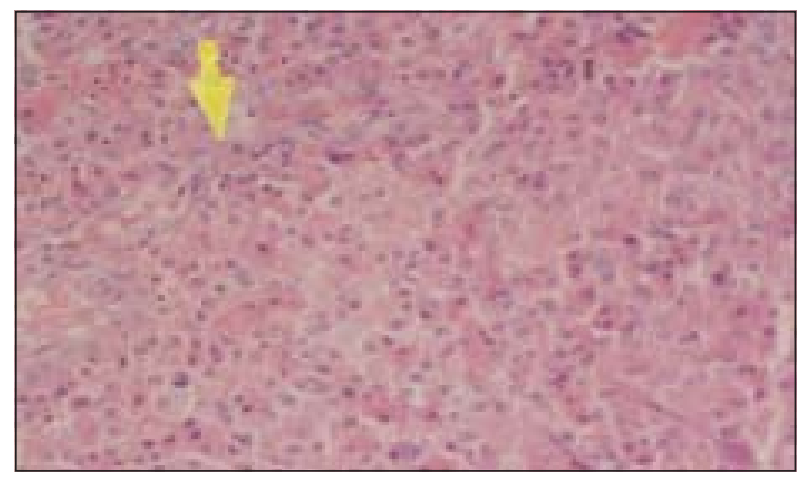

Fig.-3: Histopathological examinationshowing pituitary neoplasm composed of fairly uniform polygonal cells arranged in sheets.

\section{Discussion:}

Acromegaly is a consequence of chronic growth hormone (GH) excess, due in the majority of cases to a GH-secreting pituitary adenoma, and occurring with a population prevalence of 60 per million and an incidence of 3-4 per million per year ${ }^{1}$. Males and females appear to be equally affected with an average age of presentation of 44 years $^{1}$.

Considerable evidence suggests acromegaly can cause specific type of cardiomyopathy which can result in structural and functional abnormalities resulting in heart failure. This may be partially reversed by effective reduction in GH and IGF-1 levels ${ }^{2}$, The patient in this report had massive cardiomegaly with a cardiothoracic ratio of $<0.9$. In acromegaly, cardiac enlargement is a consistent finding and seems to be disproportionate, compared with the increase in size of other internal body organs. ${ }^{2}$ An increased frequency of systemic hypertension and premature coronary artery disease have also been described. ${ }^{2}$

Epidemiological study showed that in patients with acromegaly, valvular abnormalities are more prevalent than in control subjects, who were individually matched for left ventricular function, age, sex, and the presence of hypertension ${ }^{3}$. In addition, it has been found that in acromegalic cardiomyopathy myocardial hypertrophy with interstitial fibrosis, lymphomononulear infiltration, and areas of monocyte necrosis resembling myocarditis are prominent histopathological features. These changes often result in increased left ventricular mass and concentric hypertrophy. ${ }^{2,4}$

Left ventricular hypertrophy occurs first, often leading to slow deterioration of diastolic function early in the disease. ${ }^{5,6}$ This finding has been reported in acromegalic patients even with disease duration shorter than five years. ${ }^{7.8}$ Our patient developed overt heart failure at the age of 70 years with clinical features of acromegaly for almost seven years. Then she suddenly developed heart failure.

Clinically evident congestive heart failure may develop when the disease is untreated or unsuccessfully treated. ${ }^{7}$ Our patient sought medical attention because of severe symptoms of congestive heart failure. Data are limited on clinically evident heart failure in acromegalics. Damganovics et al reported high-output failure in $10 \%$ of patients, while Hayward et al found only 7 out of 256 patients $(<1 \%)$ with clinically evident heart failure. ${ }^{9.10}$

Echocardiographic findings in this patient showed significant increase in end-systolic and end-diastolic dimensions, reduction in the ejection fraction and fractional shorting, and reversal of the E/A ratio, in the presence of severe signs of congestive heart failure. These echocardiographic features have been documented in young acromegalics without any clinical evidence of cardiac impairment. ${ }^{7}$

Cardiac dysrhythmias have been documented in a few reports, due to the left ventricular remodeling that occur in this disease. ${ }^{11,12}$

However, our patient did not present with any form of ventricular arrythmia. In the absence of other causes of heart failure and mitral regurgitation in this case, we think that her heart failure was caused by acromegalic cardiomyopathy, although we couldn't confirm it by myocardial biopsy.

Treatment of acromeagly presenting with heart failure is aimed at removing the source of GH hyper secretion or at 
suppressing its activity along with other supportive treatment for heart failure. Somatostatin analogues are effective as first-line therapy and have a success rate of 45\%-65\%. ${ }^{13}$, 14. Besides this, surgery and local irradiation are other options of treatment. Our patient was, however, treated surgically which made a good symptomatic recovery of her heart failure.

\section{Conclusion:}

Though classically acromegaly patients commonly presents with progressive enlargement of limbs and coarsening of faces but rarely it may present with obvious heart failure caused by cardiomyopathy. So we need to look for endocrine cause of heart failure like acromegaly when there is no other overt cause of failing heart.

\section{References:}

1. I M Holdaway, C Rajasoorya .Epidemiology of acromegaly. Pituitary. 1999 Jun; 2 (1):29-41

2. Sacca L, Cittadini A, Fazio F. Growth hormone and the heart. Endocrine Rev. 1994; 15:555-573.

3. Sjoerd W. van Thiel, Alberto M. Pereira, Jonathan R. Lindner, Ferdinand Roelfsema, Ernst E. van der Wall, Hans Morreau et al. Increased Prevalence of Regurgitant Valvular Heart Disease in Acromegaly. Journal of Clinical Endocrinology \& Metabolism 2004:89(1):71-75

4. Frustaci A, Chimenti C, Setoguchi M, et al.Cell death in acromegalic cardiomyopathy. Circulation. 1999;99:14261434.
5. Bertoni PD, Morandi G. Impaired left ventricular diastolic function in acromegaly:an echocardiographic study. Acta Cardiol.1998;42:1-10.

6. Colao A, Cuocolo A, Marzullo P, et al. Impact of patient's age and disease duration on cardiacperformance in acromegaly: a radionuclideangiography study. J Clin Endocrinol Metab. 1999; 84: 1518-1523.

7. Colao A, Merola B, Ferone D, Lombardi G. Acromegaly. J Clin Endocrinol Metab.1997;82:2777-2781.

8. olao A, Baldelli R, Marzullo P, et al. Systemic hypertension and impaired glucose tolerance are independently correlated to the severity of acromegalic cardiomyopathy. J Clin Endocrinol Metab. 2000;85:193-199.

9. Damjanovics SS, Neskovic AN, Petakov MS, et al. High output heart failure in patients with newly diagnosed acromegaly. Am J Med. 2002;112:610-616.

10. Hayward RP, Emanuel RW, Nabarro JDN. Acromegalic heart disease: influence of treatment of acromegaly on the heart. Q J Med. 1987;62:41-58.

11. Rodrigues EA, Caruana MP, Lahiri A, Nabarro JDN, Jacobs HS, Raftery EB. Subclinical cardiac dysfunction in acromegaly: evidence for a specific disease of heart muscle. British Heart J. 1989;62:185-194.

12. Kahaly G, Olshausen KV, Mohr-Kahaly S, et al. Arrythmia profile in acromegaly. Eur Heart J. 1992;13:51-56.

13. Abs R, Verhelst J, Maiter D, et al. Carbergoline in the treatment of acromegaly: a study in 64 patients. J Clin Endocrinol Metab. 1997; 82:518-523.

14. Chanson P, Leselbaum A, Blumberg J, et al. Efficacy and tolerability of long-acting somatostatin analogue lanreotide in acromegaly.A 12-month multicenter study of 58 acromegalic patients. Pituitary. 2000; 2:269-276. 